\title{
Upregulation of endothelial cell-derived exosomal microRNA-125b-5p protects from sepsis-induced acute lung injury by inhibiting topoisomerase II alpha
}

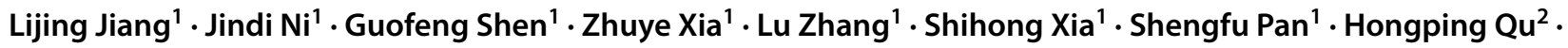 \\ Xiang $\mathrm{Li}^{1}{ }^{1}$
}

Received: 13 April 2020 / Revised: 10 October 2020 / Accepted: 20 October 2020 / Published online: 2 January 2021

(c) Springer Nature Switzerland AG 2021

\begin{abstract}
Objective Emerging evidence has revealed that exosomal microRNAs (miRNAs) are implicated in human diseases. However, role of exosomal miR-125b-5p in sepsis-induced acute lung injury (ALI) remains further explored. We focused on the effect of exosomal miR-125b-5p on ALI progression via targeting topoisomerase II alpha (TOP2A).

Methods The ALI mouse models were established by cecal ligation and perforation, which were then treated with miR125b-5p agomir or overexpressed TOP2A. Next, the pathological structure of ALI mouse lung tissues were observed, miR125b-5p, TOP2A and vascular endothelial growth factor (VEGF) expression was determined, and the lung water content, inflammatory response, protein content in bronchoalveolar lavage fluid (BALF) and cell apoptosis in ALI mouse lung tissues were assessed. Exosomes were extracted from endothelial cells (ECs) and identified, which were then injected into the modeled mice to observe their roles in ALI. The targeting relationship between miR-125b-5p and TOP2A was confirmed. Results MiR-125b-5p was downregulated while TOP2A was upregulated in ALI mice. MiR-125b-5p elevation or ECsderived exosomes promoted VEGF expression, improved pathological changes and restrained lung water content, inflammatory response, protein content in BALF and cell apoptosis in lung tissues ALI mice. TOP2A overexpression reversed the repressive role of miR-125b-5p upregulation in ALI, while downregulated miR-125b-5p abrogated the effect of ECs-derived exosomes on ALI. TOP2A was confirmed as a direct target gene of miR-125b-5p.

Conclusion Our study indicates that ECs-derived exosomes overexpressed miR-125b-5p to protect from sepsis-induced ALI by inhibiting TOP2A, which may contribute to ALI therapeutic strategies.
\end{abstract}

Keywords Sepsis-induced acute lung injury $\cdot$ Endothelial cells-derived exosome $\cdot$ MicroRNA-125b-5p · Topoisomerase II alpha $\cdot$ Vascular endothelial growth factor

Responsible Editor: John Di Battista.

Lijing Jiang, Jindi Ni are first co-author.

Xiang Li

LIxiang469@163.com

1 Department of Critical Care Medicine, Minhang Hospital, Fudan University, No. 39, Xinling Road, Minhang District, Shanghai 201199, China

2 Department of Critical Care Medicine, Ruijin Hospital, Shanghai Jiao Tong University School of Medicine, Shanghai 200025, China

\section{Introduction}

As described in the Third International Consensus Definitions for Sepsis and Septic Shock, sepsis is an organ dysfunction that threatens life and results from a dysregulated host response to infection [1]. Organ failure is one of the most serious complications of sepsis and lung is one of the first organs to fail, which could not be resolved quickly. Additionally, the lung failure in sepsis may progress to acute lung injury (ALI). Indeed, about $50 \%$ of sepsis patients will get ALI and acute respiratory distress syndrome (ARDS) [2]. ALI is a syndrome featured by hypoxemia, widespread capillary leakage, low lung compliance and noncardiogenic pulmonary edema [3]. The pathology of sepsis-induced ALI remains less explored. Sepsis and 
ALI patients could only be treated with antibiotics and supportive measures, while these treatments have limited effects on the high mortality [4]. Thus, it is urgent to investigate more efficient targets to decelerate the progression of sepsis-induced ALI.

MicroRNAs (miRNAs) are non-coding RNAs with 21-25 nucleotides that usually inhibit the translation and stability of mRNAs and control genes. MiRNAs are able to modulate protein expression via binding to $3^{\prime}$-untranslation region ( $3^{\prime}$-UTR) in the target mRNA [5]. Some particular miRNAs, such as miR-145 [6] and miR-206 [7] have been revealed to be involved in the development of sepsis-induced ALI. miR-125b-5p has been verified to be abnormally expressed in sepsis [8] and impact of miR-125b on lipopolysaccharide (LPS)-induced ALI has been previously studied [9], while role of miR-125b-5p in the development of sepsis-induced ALI has not been explored yet. Topoisomerase II alpha (TOP2A) is situated at chromosome 17q12-q21 and is a critical nuclear enzyme regulating topological states of DNA. Thus, TOP2A is implicated in processes such as DNA replication and transcription [10]. Although TOP2A has been identified as an essential potential target for pediatric sepsis [11] and sepsis-related ARDS [12], its role in sepsisinduced ALI and the target relation between miR-125b-5p and TOP2A remains unexplored. Furthermore, exosomes are the smallest extracellular vesicles with a diameter of $30-100 \mathrm{~nm}$ that can be secreted by almost all cells and contain mRNA and short RNAs, including miRNAs [13]. Therefore, exosomes are able to transfer miRNAs to exert regulatory effects on human diseases. For instance, it has been revealed that endothelial progenitor cell-derived exosomes could attenuate ALI via transferring miR-126 [14], and miR-30b-3p-overexpressing mesenchymal stem cells (MSC)-released exosomes were demonstrated to alleviate LPS-induced ALI [15]. Therefore, we aim to testify the role of exosomal miR-125b-5p in sepsis-induced ALI progression by targeting TOP $2 \mathrm{~A}$, and we supposed that the exosomes and elevated miR-125b-5p may protect against sepsis-induced ALI by inhibiting TOP2A.

\section{Materials and methods}

\section{Ethics statement}

Animal experiments were strictly in accordance with the Guide to the Management and Use of Laboratory Animals issued by the National Institutes of Health. The protocol of animal experiments was approved by the Institutional Animal Care and Use Committee of Minhang Hospital, Fudan University.

\section{Extraction of endothelial cell-derived exosomes (EC-Exos)}

The cerebral microvascular endothelial cells (b END.3) that purchased from Shanghai enzyme research Biotechnology Co., Ltd. (Shanghai, China) were cultured in Dulbecco's modified Eagle medium (DMEM) containing 10\% phosphate-buffered saline (PBS), 1\% L-glutamine, $100 \mathrm{U} /$ $\mathrm{mL}$ penicillin and $100 \mathrm{mg} / \mathrm{mL}$ streptomycin until the cell confluence reached $80-90 \%$. The cell supernatant was conducted with ultracentrifugation to extract exosomes: $4{ }^{\circ} \mathrm{C}$ and $300 \mathrm{~g}$ for $10 \mathrm{~min}, 800 \mathrm{~g}$ for $15 \mathrm{~min}, 10,000 \mathrm{~g}$ for $30 \mathrm{~min}$ with the supernatant collected in each centrifugation, then centrifuged at $100,000 \mathrm{~g}$ for $70 \mathrm{~min}$ with the supernatant discarded. The sediment was resuspended to obtain the external vesicles, which were preserved at $-80^{\circ} \mathrm{C}$.

\section{Identification of EC-Exos}

EC-Exo morphology was observed through a transmission electron microscopy (TEM): $20 \mu \mathrm{L}$ exosome suspension was added onto a copper network (bore diameter at $2 \mathrm{~nm}$ ) for $3 \mathrm{~min}$, which was then added with $30 \mu \mathrm{L} 3 \%$ phosphotungstic acid solution for $5 \mathrm{~min}$, and the morphology of exosomes was observed and photographed under a TEM.

\section{Lentivirus infection of ECs}

ECs were respectively infected with lentivirus that loaded with inhibited miR-125b-5p or its negative control (NC) to obtain EC-miR-125b-5p antagomir and EC-antagomir NC. ECs were cultured for $48 \mathrm{~h}$ to collect the supernatant. The lentiviruses were obtained from GenePharma Co., Ltd., (Shanghai, China). Relative Exos (Exos-antagomir $\mathrm{NC}$ and Exos-miR-125b-5p antagomir) were extracted by ultracentrifugation.

\section{Experimental animals}

A number of 122 healthy specific pathogen-free BABL/c mice (aged 6-8 w, weighed 18-22 $\mathrm{g}$ and in half gender) were acquired from Beijing Vital River Laboratory Animal Technology Co., Ltd. (Beijing, China).

\section{Establishment of ALI mice models}

The ALI mice models were established by cecal ligation puncture (CLP) [16]: the mice were fasted for $8 \mathrm{~h}$ before the surgery and then anesthetized by intraperitoneal injection of $1 \%$ pentobarbital sodium $(80 \mathrm{mg} / \mathrm{kg})$. Next, the mice were fixed with a supine position with their abdomen disinfected 
and with a longitudinal incision $(1.0 \mathrm{~cm})$ at the middle section of linea alba. The cecum was carefully taken out of the abdomen and $1 / 2$ of the cecum was ligated by 1-0 suture, and the contralateral margin of mesentery was punched with some intestinal contents squeezed out, then the cecum was put back into the abdomen and the incision was sutured. Normal saline $(1 \mathrm{~mL})$ was subcutaneously injected into mice after surgery, and then the mice were normally fed in single cages.

\section{Grouping of lentivirus-treated mice}

Except for the sham-operated mice (conducted with laparotomy but not CLP), other mice were conducted with CLP for sepsis-induced ALI $1 \mathrm{~h}$ after tracheal instillation of 20 $\mu \mathrm{L}$ PBS, $20 \mu \mathrm{L}$ miR-125b-5p agomir NC (200 nM), $20 \mu \mathrm{L}$ miR-125b-5p agomir (200 nM), $20 \mu \mathrm{L}$ miR-125b-5p agomir NC_+overexpressed (OE)-TOP2A $(200 \mathrm{nM})$, as well as $20 \mu \mathrm{L}$ miR-125b-5p agomir + OE-TOP2A $(200 \mathrm{nM})(n=6$ mice).

\section{Grouping of EC-Exos-treated mice}

As for the grouping of EC-Exos-treated mice, the shamoperated mice were conducted with laparotomy but not CLP, while the other mice were conducted with CLP for sepsisinduced ALI $1 \mathrm{~h}$ after tracheal instillation of $20 \mu \mathrm{L}$ PBS, $80 \mu \mathrm{g}$ Exos, $80 \mu \mathrm{g}$ Exos-antagomir NC and $80 \mu \mathrm{g}$ Exos-miR$125 b-5 p$ antagomir (Exos were resuspended in $20 \mu \mathrm{L}$ PBS).

\section{Tracheal instillation}

Mice were intraperitoneally anesthetized by $1 \%$ pentobarbital sodium and fixed with a supine position $\left(45^{\circ}\right)$ to keep airway patency; then the tongue was moved to one side of the oral cavity. Next, the neck skin was disinfected and cut open to expose the trachea. The mixture of Exos or lentiviral vector was instilled into the trachea by a microsyringe, and the mice were held up and rotated after the instillation to make the mixture fully distribute in lung. The breath and heartbeat of the mice were observed during the instillation, which was immediately stopped if there appeared a cardiorespiratory arrest. After the trachea was unobstructed and the breath and heartbeat were stabilized, the instillation was continued.

\section{Sample treatment and bronchoalveolar lavage fluid (BALF) collection}

The mice were intraperitoneally anesthetized by $1 \%$ pentobarbital sodium $24 \mathrm{~h}$ after the treatment and $3 \mathrm{~mL}$ blood was extracted from the abdominal aorta. The blood was centrifuged at $4^{\circ} \mathrm{C}$ and $3,000 \mathrm{r} / \mathrm{min}$ for $10 \mathrm{~min}$ and the supernatant was collected and preserved at $-20{ }^{\circ} \mathrm{C}$. Afterwards, the mice were euthanized with their lung tissues harvested. A part of the lower lobes of right lung were preserved in $10 \%$ neutral formalin for pathological and apoptosis examinations. After the wet weight was measured, the middle lobes were immediately toasted at $56{ }^{\circ} \mathrm{C}$ for $72 \mathrm{~h}$ and the dry weight was evaluated; then the rate of wet weight and dry weight (W/D) was calculated. The upper lobes were preserved in liquid nitrogen for expression evaluation. The left lung tissues were washed with $0.4 \mathrm{~mL}$ normal saline with a $1-\mathrm{mL}$ injector for three times and $1 \mathrm{~mL}$ BALF was collected. The BALF was then centrifuged at $4^{\circ} \mathrm{C}$ and $1,500 \mathrm{rpm}$ for $5 \mathrm{~min}$ and preserved at $-80{ }^{\circ} \mathrm{C}$ with the supernatant discarded.

\section{Bicinchoninic acid (BCA) assay}

The microplate reader was preheated $15 \mathrm{~min}$ before the measurement, and then the protein contents in BALF were evaluated in line with the instruction of the BCA kit (P0010, Beyotime Institute of Biotechnology, Shanghai, China). The optical density (OD) value at $570 \mathrm{~nm}$ was analyzed using the microplate reader. With the concentration of relative standard taken as the ordinate, the standard curve was phased, and the protein concentrations in BALF were calculated based on OD values of the samples by the standard curve.

\section{Enzyme-linked immunosorbent assay (ELISA)}

The concentrations of tumor necrosis factor- $\alpha$ (TNF- $\alpha$ ), interleukin (IL)- $1 \beta$ and IL-6 in BALF, and the concentration of vascular endothelial growth factor (VEGF) in BALF and serum were determined based on the directions of ELISA kits (R\&D Systems, Minneapolis, MN, USA). The OD value at $490 \mathrm{~nm}$ was measured, and then the concentrations of TNF- $\alpha$, IL-1 $\beta$, IL-6 and VEGF were calculated.

\section{Hematoxylin-eosin (HE) staining}

HE staining of the lower lobes of the right lungs was performed as previously described [7]. The tissues were observed and photographed under a microscope.

\section{Terminal deoxynucleotidyl transferase-mediated deoxyuridine triphosphate nick end-labeling (TUNEL) staining}

The tissues were normally embedded, sectioned, dewaxed, and stained based on the protocols of TUNEL kits (Roche Ltd., Basel, Switzerland): the sections were successively incubated with $100 \mu \mathrm{L}$ proteinase $\mathrm{K}$ for $15 \min , 50 \mu \mathrm{L}$ TUNEL reaction solution for $1 \mathrm{~h}, 0.3 \% \mathrm{H}_{2} \mathrm{O}_{2}$ for $20 \mathrm{~min}$ and horseradish peroxidase (HRP)-conjugated streptavidin for $30 \mathrm{~min}$, and then stained by diaminobenzidine. After 
counterstaining by hematoxylin, the sections were routinely dehydrated, permeabilized and sealed. The apoptotic cells (TUNEL positive cells) were stained into brownish-yellow under a light microscope. Sections in different groups were randomly selected and photographed.

\section{Reverse transcription quantitative polymerase chain reaction ( $R T-q P C R)$}

Total RNA in tissue was extracted using Trizol kits (Invitrogen, CA, USA), and the reverse transcription was performed using high-power capacity cDNA reverse transcription kits and TaqMan MicroRNA reverse transcription kits (Thermo Fisher Scientific, MA, USA). The PCR was conducted by the ABI 7300 system (Thermo Fisher Scientific) and SYBR Premix Ex Taq (TaKaRa Biotechnology Co., Ltd., Liaoning China). The primers are shown in Table 1 and data were analyzed by $2^{-\Delta \Delta \mathrm{Ct}}$ method.

\section{Western blot analysis}

Total protein in the lung tissues of mice was extracted by cell lysis buffer kits (P0013B, Beyotime) for the measurement of protein levels of TOP2A and VEGF. The mice serum was appended with extraction buffer and centrifuged at $4^{\circ} \mathrm{C}$ and $14,000 \mathrm{rpm}$ for $10 \mathrm{~min}$. The supernatant was used for the assessment of protein levels of exosome marker proteins (CD63, HSP70 and TSG101). The BCA kits (P0010, Beyotime) were used to determine the protein concentrations: the proteins were conducted with $10 \%$ sodium dodecyl sulfate-polyacrylamide gel electrophoresis for $2 \mathrm{~h}$ and transferred onto membranes, which were blocked with 5\% skim milk powder for $2 \mathrm{~h}$ and supplemented with $4 \mathrm{~mL}$ primary antibodies TOP2A (1: 100), VEGF (1: 1000), $\beta$-actin (1: 500, all from Santa Cruz Biotechnology Inc, CA, USA), CD63 (1: 800), HSP70 (1: 1000) and TSG101 (1: 3000, all from Abcam Inc.,
Cambridge, MA, USA) and incubated at $4{ }^{\circ} \mathrm{C}$ overnight. Subsequently, the membranes were added with $4 \mathrm{~mL} \mathrm{sec-}$ ondary antibody goat anti-rabbit immunoglobulin G/HRP and incubated for $1 \mathrm{~h}$, then exposed and developed. With $\beta$-actin as the loading control, the gray values were analyzed by the Image Lab software and protein expression was calculated. The measurement of protein expression of TOP2A, VEGF, CD63, HSP70 and TSG101 in ECs of mice was same as above.

\section{Dual luciferase reporter gene assay}

The target relation and binding sites of miR-125b-5p and TOP2A were predicted by an online prediction software (https://cm.jefferson.edu/). The wild-type (WT) or mutation type (MUT) segments of miR-125b-5p and TOP2A were subcloned into pGL3 luciferase reporter vector (Promega, Beijing, China), and the plasmids were co-transfected with miR-125b-5p mimics or its NC into 293 T cells for $48 \mathrm{~h}$. The luciferase activity was evaluated using a dual luciferase reporter gene detection system (Promega).

\section{Statistical analysis}

All data analyses were conducted using SPSS 21.0 software (IBM Corp. Armonk, NY, USA). The measurement data conforming to the normal distribution were expressed as mean \pm standard deviation. The unpaired t-test was performed for comparisons between two groups, one-way analysis of variance (ANOVA) was used for comparisons among multiple groups and the Tukey's post hoc test was used for pairwise comparisons after one-way ANOVA. $P$ value $<0.05$ was indicative of statistically significant difference.
Table 1 Primer sequence

\begin{tabular}{ll}
\hline Gene & Primer sequence (5'-3') \\
\hline MiR-125b-5p & Forward: 5'-GTGCTAGCACTGGACCACCTGTTTGC-3' \\
& Reverse: 5'-GTCTGCAGAAGGGTGTATTACCATCACTTC-3' \\
U6 & Forward: 5'-TTAGCATGGCCCCTGC-3' \\
& Reverse: 5'-TGCGTGTCGTGGAGTC-3' \\
TOP2A & Forward: 5'-GCCATTGGCTGTGGTATTG-3' \\
& Reverse: 5'-GAGAAGCTTCTCGAACATTGAG-3' \\
VEGF & Forward: 5'-ACATTGGCTCACTTCCAGAAACAC-3' \\
& Reverse: 5'-GGTTGGAACCGGCTCATCTTTATC-3' \\
$\beta$-actin & Forward: 5'-CATCCGTAAAGACCTCTATGCCAAC-3' \\
& Reverse: 5'-ATGGAGCCACCGATCCACA-3' \\
\hline
\end{tabular}

MiR-125b-5p microRNA-125b-5p, TOP2A topoisomerase II alpha, VEGF vascular endothelial growth factor 


\section{Results}

\section{Observation of mice after CLP treatment}

Sepsis is a life-threatening disease resulted from a dysregulated host response to infection and is a common complication of critical postoperative patients. The lung is one of the most frequently affected organs during sepsis. About $50 \%$ of patients with sepsis develop ALI or acute respiratory distress syndrome [17]. In order to study the sepsis-induced ALI, in this study, the model was established by CLP in mice and the general manifestations of the mice were observed.

The mice woke up 2-3 h after CLP; sham-operated mice had normal condition and routine action and diet, and there was no pus exuded from their abdomen and no obvious swelling in organs. In mice underwent CLP, or further treated with agomir NC, or miR-125b-5p agomir + OETOP2A, the anesthesia recovery period was prolonged and there appeared breathlessness, faint consciousness, hair standing shiver and death, and the mortality reached $50 \%$ in $24 \mathrm{~h}$. By a laparotomy, sepsis abdominal infection symptoms such as swelled intestine, purulent seroperitoneum, turbidity, and cacosmia could be found. Treatment of miR-125b-5p agomir in mice improved the condition of mice with sepsis-induced ALI while the improvement was reversed by OE-TOP2A. The infection was especially serious in mice that underwent CLP and were treated with agomir NC+ OE-TOP2A.

\section{miR-125b-5p and VEGF are reduced while TOP2A is elevated in lung tissues of ALI mice}

A study has validated that miR-125b upregulation could attenuate LPS-induced ALI [9]. In the early stage of ALI, alveolar macrophages and type II epithelial cells produce a large amount of VEGF temporarily, resulting in vascular leakage and interstitial edema [18]. miR-125b-5p, VEGF and TOP2A expression in lung tissues was determined. The results (Fig. 1a-c) indicated that CLP mice had downregulated miR-125b-5p and VEGF expression and upregulated TOP2A expression. miR-125b-5p agomir elevated miR$125 b-5 p$ and VEGF expression and reduced TOP2A expression. miR-125b-5p agomir reversed the effect of OE-TOP2A on miR-125b-5p, VEGF and TOP2A expression.

\section{Elevated miR-125b-5p restricts W/D value in lung tissues and protein content in BALF in ALI mice}

The W/D values in lung tissues and protein content in BALF of the mice were measured. The results (Fig. 2a, b) reflected that CLP mice had increased W/D values and protein content in BALF, and the increase of W/D values and protein content in BALF could be attenuated by miR-125b-5p agomir. miR$125 b-5 p$ agomir reversed OE-TOP2A-induced elevation in $\mathrm{W} / \mathrm{D}$ values and protein content in BALF in ALI mice.

\section{Elevated miR-125b-5p restrains inflammatory response in BALF and regulates VEGF expression in BLAF and serum of ALI mice}

Moreover, it has been elucidated that the decrease of VEGF production caused by apoptosis of bronchial epithelial cells could be hydrolyzed and degraded by neutrophils-released proteinase, and VEGF was transferred from BALF to circulation, resulting in the increase of VEGF content in serum [19]. Next, levels of TNF- $\alpha$, IL- $1 \beta$ and IL- 6 in BALF and VEGF expression in BALF and serum were measured by ELISA. The outcomes indicated that (Fig. 2c, d) levels of TNF- $\alpha$, IL- $1 \beta$, IL- 6 in BALF and VEGF expression in serum were enhanced, while VEGF expression was decreased in BALF of ALI mice, and these alterations were reversed by
A

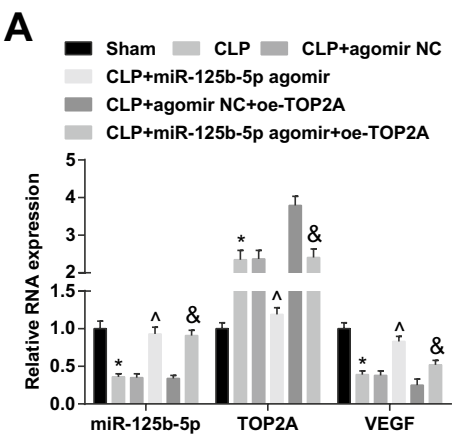

B

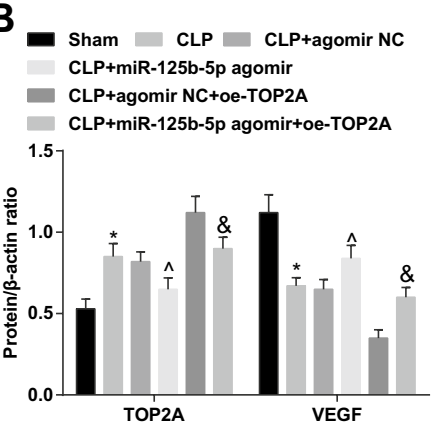

C

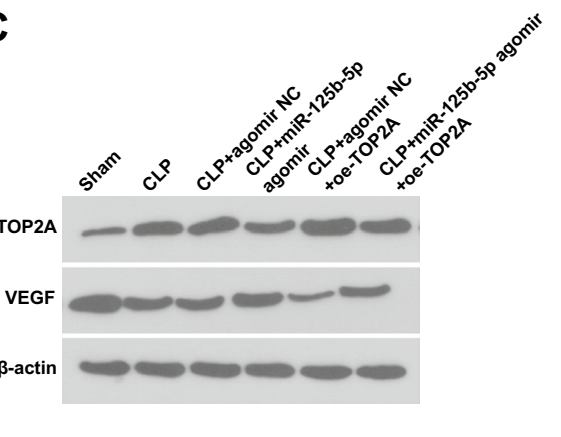

Fig. $1 \mathrm{miR}-125 \mathrm{~b}-5 \mathrm{p}$ and VEGF are reduced while TOP2A is elevated in lung tissues of ALI mice. a miR-125b-5p expression, and mRNA expression of TOP2A and VEGF in lung tissues of mice; $\mathbf{b}$ protein expression of TOP2A and VEGF in lung tissues of mice; $\mathbf{c}$ protein bands of TOP2A and VEGF in lung tissues of mice; $* P<0.05$ vs the sham group, ${ }^{\wedge} P<0.05$ vs the CLP + agomir NC group, ${ }^{\&} P<0.05$ vs the CLP + agomir NC+OE-TOP2A group. The measurement data were expressed as mean \pm standard deviation, one-way ANOVA was used for comparisons among multiple groups and Tukey's post hoc test was used for pairwise comparisons after one-way ANOVA 
Fig. 2 Elevated miR-125b-5p restricts $\mathrm{W} / \mathrm{D}$ value in lung tissues and protein content in BALF of ALI mice and also restrains inflammatory response in BALF and regulates VEGF expression in BALF and serum of ALI mice. a W/D values in lung tissues of mice; $\mathbf{b}$ protein contents in BALF; c concentrations of TNF- $\alpha$, IL- $1 \beta$ and IL-6 in BALF; $\mathbf{d}$ VEGF expression in BALF and serum; $* P<0.05$ vs the sham group, ${ }^{\wedge} P<0.05$ vs the CLP + agomir NC group, ${ }^{\&} P<0.05$ vs the CLP + agomir NC+OE-TOP2A group. The measurement data were expressed as mean \pm standard deviation, one-way ANOVA was used for comparisons among multiple groups and Tukey's post hoc test was used for pairwise comparisons after one-way ANOVA
A
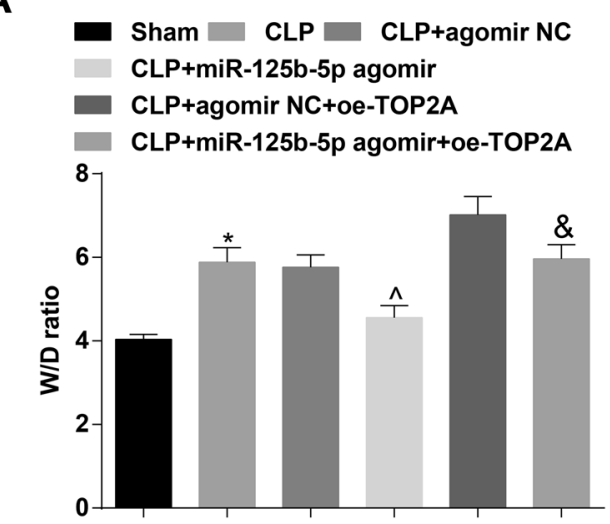

C

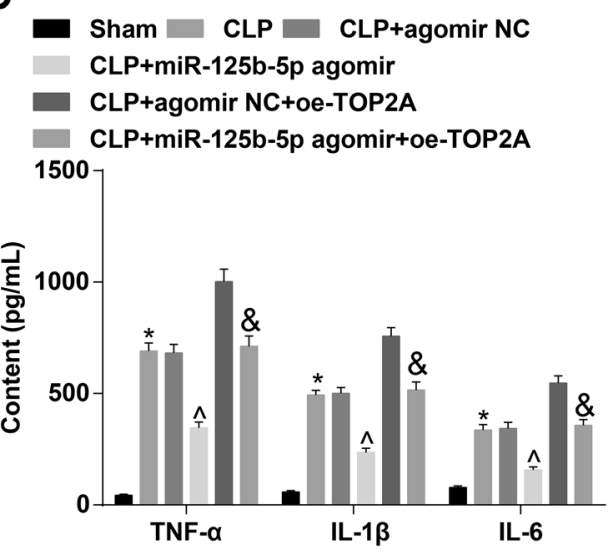

B

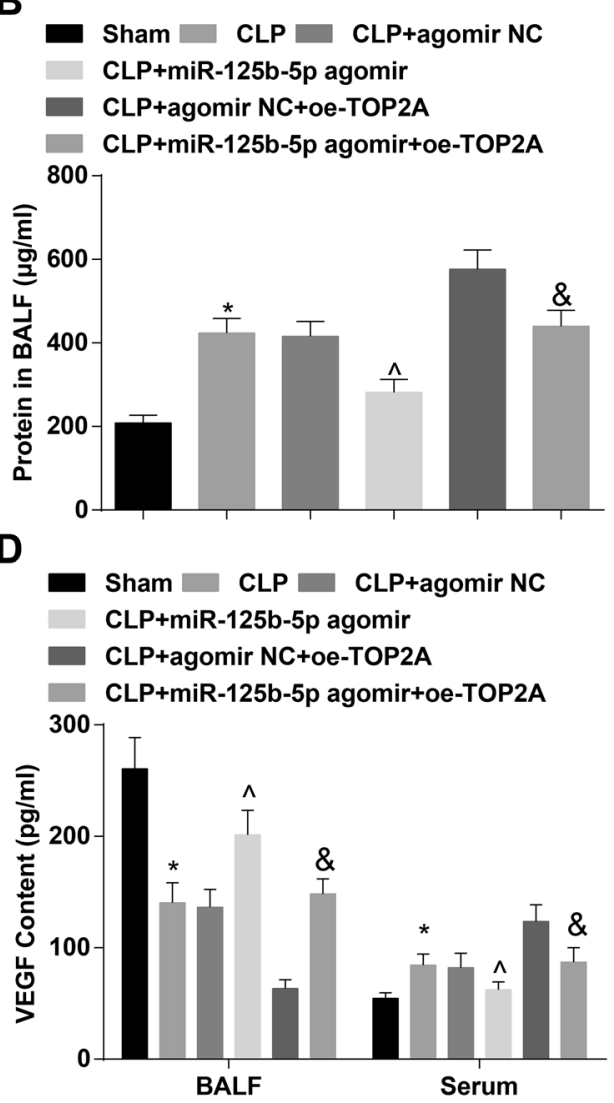

miR-125b-5p agomir. Effect of OE-TOP2A on TNF- $\alpha$, IL-1 $\beta$ and IL-6 levels in BALF and VEGF expression in BALF and serum were reversed by miR-125b-5p agomir.

\section{Elevated miR-125b-5p improves pathological changes and decelerates cell apoptosis in lung tissues of ALI mice}

The pathological structure of mouse lung tissue was assessed and as observed through HE staining (Fig. 3a), the alveolus structure in mouse lung tissues in sham-operated,oce was integrate, and there were no obvious inflammatory cell infiltration and congestion in blood capillary. In mice underwent CLP, or further treated with agomir NC, or miR-125b-5p agomir + OE-TOP2A, there were serious inflammation, disordered and blurred alveolus structure, fractured alveolar septum, inflammatory cell infiltration and abundant erythrocytes in blood capillary. In mice treated with miR-125b-5p agomir, the lung injury was attenuated, and there showed approximate alveolar structure and less inflammatory cell infiltration in alveoli and pulmonary mesenchyme. Mice underwent CLP and treated with agomir NC+ OE-TOP2A had the most serious inflammation reaction.

In TUNEL staining, the TUNEL positive cells represented for the apoptotic cells, and the outcomes suggested that (Fig. 3b) the ALI mice had more apoptotic cells and this increase was abolished by miR-125b-5p agomir. miR$125 \mathrm{~b}-5 \mathrm{p}$ agomir reversed impact of OE-TOP2A on cell apoptosis in the lung tissues of ALI mice.

\section{Identification of EC-Exos}

Exosomes were extracted from epithelial cells and identified. Through a TEM, it could be observed that the diameter of EC-Exos ranged from 40 to $100 \mathrm{~nm}$, and the exosomes were round or oval (Fig. 4a). The expression of exosome marker proteins was assessed and the results (Fig. 4b) reflected that the exosome marker proteins CD63, HSP70 and TSG101 were all expressed in the extracted products of our experiment.

\section{EC-Exos up-regulate miR-125b-5p and VEGF while down-regulate TOP2A in lung tissues of $A L I$ mice}

miR-125b-5p, VEGF and TOP2A expression was determined and we found that (Fig. 4c-e) CLP induction in mice decreased miR-125b-5p and VEGF expression and increased TOP2A expression, and these alterations were reversed by EC-Exos. Exosomes carrying miR-125b-5p antagomir 


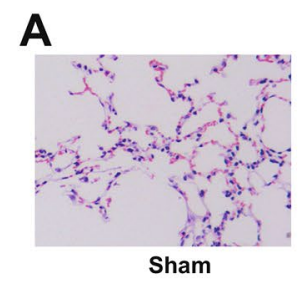

B
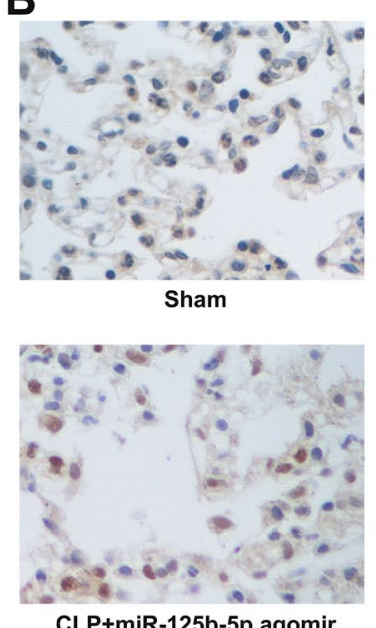

CLP+miR-125b-5p agomir

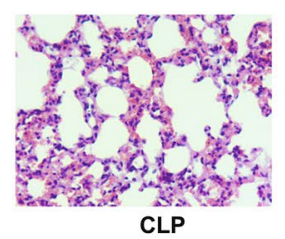

CLP

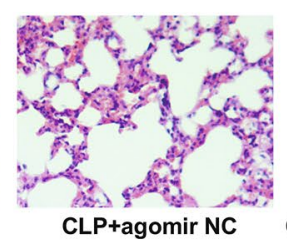

CLP+agomir NC

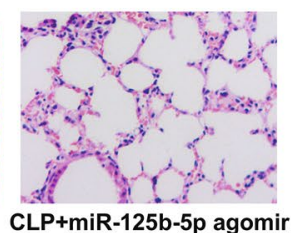

CLP+miR-125b-5p agomir

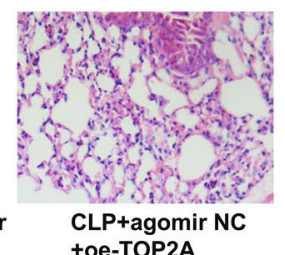

+oe-TOP2A
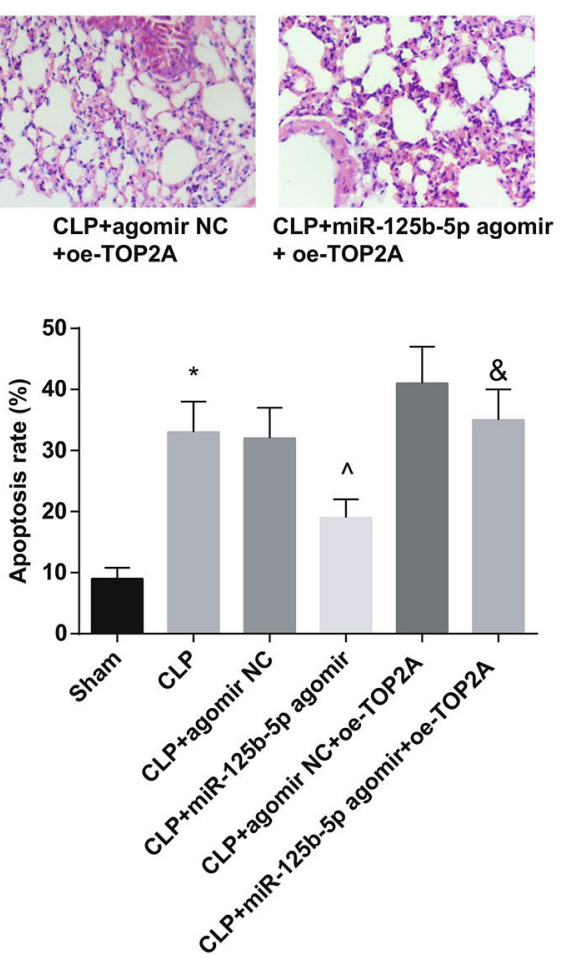

Fig. 3 Elevated miR-125b-5p improves pathological changes and decelerates cell apoptosis in lung tissues of ALI mice. a pathological structure of lung tissues of mice; $\mathbf{b}$ representative images of TUNEL staining in lung tissues of mice and TUNEL positive cells in lung tissues of mice; ${ }^{*} P<0.05$ vs the sham group, ${ }^{\wedge} P<0.05$ vs the CLP+agomir NC group, ${ }^{\&} P<0.05$ vs the CLP+agomir $\mathrm{NC}+\mathrm{OE}-\mathrm{TOP} 2 \mathrm{~A}$ group. The measurement data were expressed as mean \pm standard deviation, one-way ANOVA was used for comparisons among multiple groups and Tukey's post hoc test was used for pairwise comparisons after one-way ANOVA restrained miR-125b-5p and VEGF expression but promoted TOP2A expression.

\section{EC-Exos reduce W/D value in lung tissues and protein content in BALF of ALI mice}

Subsequently, measurement of W/D value in lung tissues and protein content in BALF in mice was performed (Fig. 5a, b) and the outcomes mirrored that EC-Exos suppressed W/D values in lung tissues and protein content in BALF that enhanced by CLP; EC-Exos conveying miR-125b-5p antagomir promoted the W/D values in lung tissues and protein content in BALF of ALI mice.

\section{EC-Exos restrain inflammatory response in BALF and regulate VEGF expression in BALF and serum of ALI mice}

Concentrations of TNF- $\alpha$, IL- $1 \beta$, IL- 6 and VEGF were determined and results of ELISA (Fig. 5c, d) reflected that levels of TNF- $\alpha$, IL- $1 \beta$ and IL- 6 in BALF and VEGF expression in serum were heightened, while VEGF expression in BALF was reduced in ALI mice. EC-Exos treatment reduced levels of TNF- $\alpha$, IL- $1 \beta$ and IL- 6 in BALF, elevated VEGF expression in BALF and promoted VEGF expression in serum. EC-Exos conveying miR-125b-5p antagomir resulted in an increase in levels of TNF- $\alpha$, IL- $1 \beta$ and IL- 6 in BALF and VEGF expression in serum, and a reduction of VEGF expression in BALF.

\section{EC-Exos improve pathological changes and decelerate cell apoptosis in lung tissues of $A L I$ mice}

After that, the lung tissue samples in each group of mice were subjected to HE staining to observe the structure of lung tissues. According to the outcomes of HE staining (Fig. 6a), the alveolus structure in mouse lung tissues in sham-operated mice was integrate, and there were no obvious inflammatory cell infiltration and congestion in blood capillary. In mice underwent CLP or further treated with Exos-miR-125b-5p antagomir, there were serious inflammation, disordered and blurred alveolus structure, fractured alveolar septum, inflammatory cell infiltration and abundant erythrocytes in blood capillary. In mice underwent CLP or further treated with Exos or 

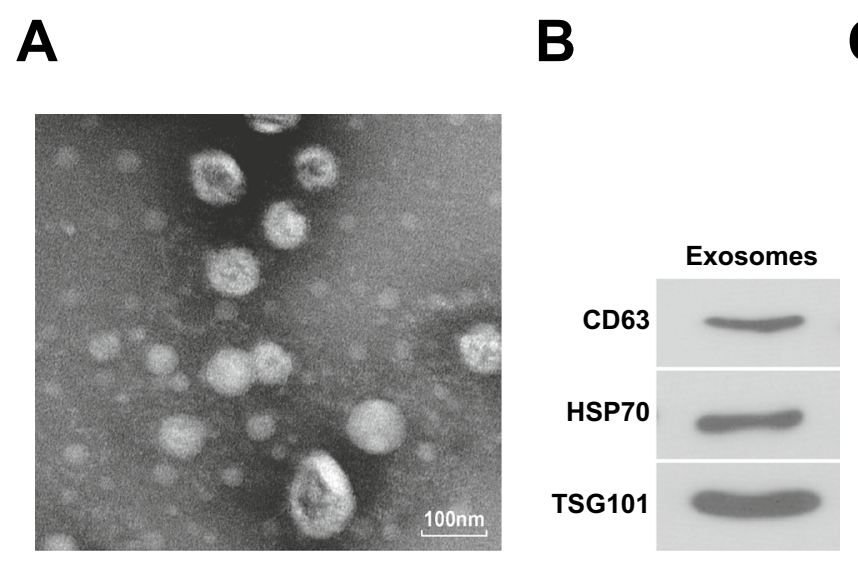

C
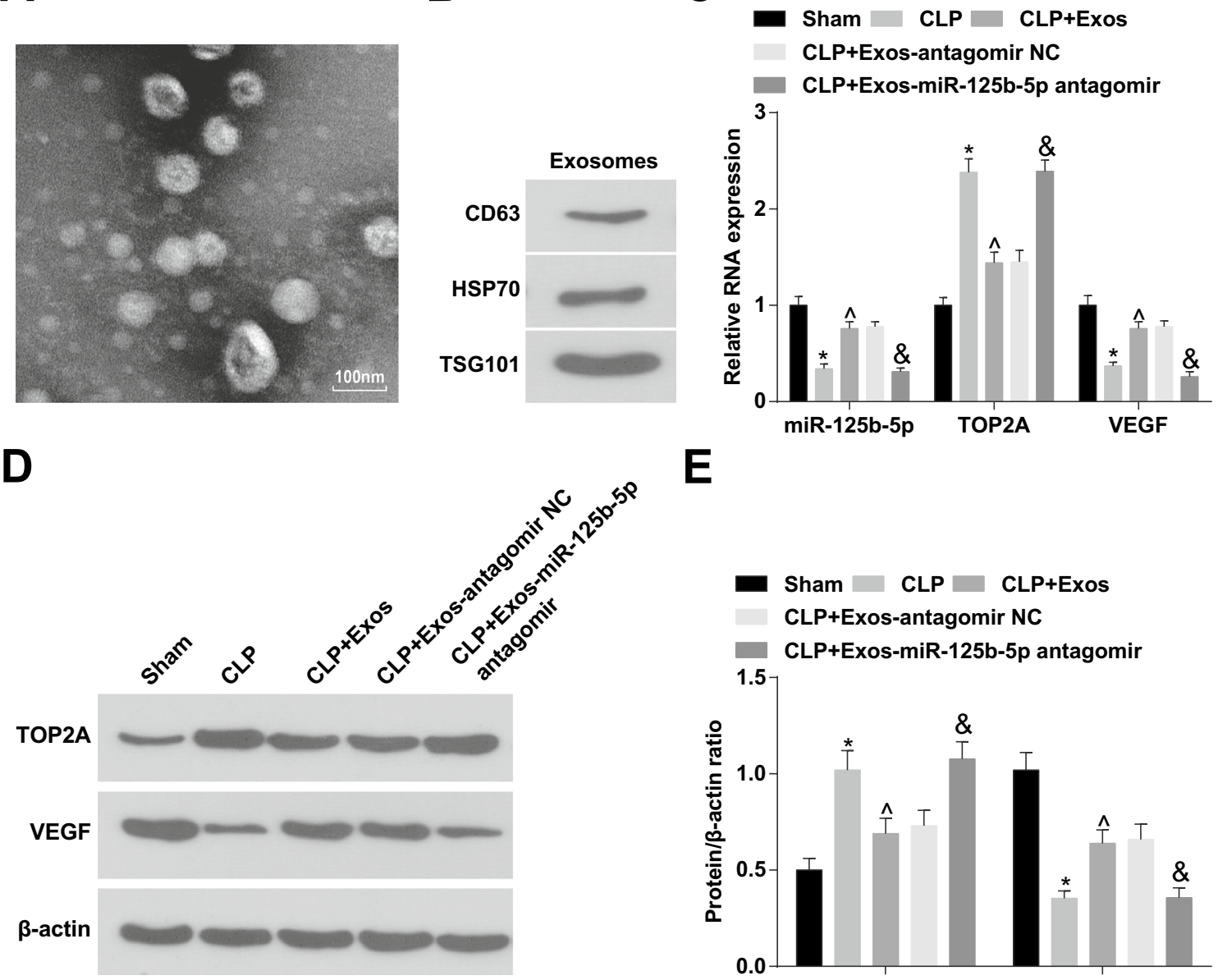

$E$

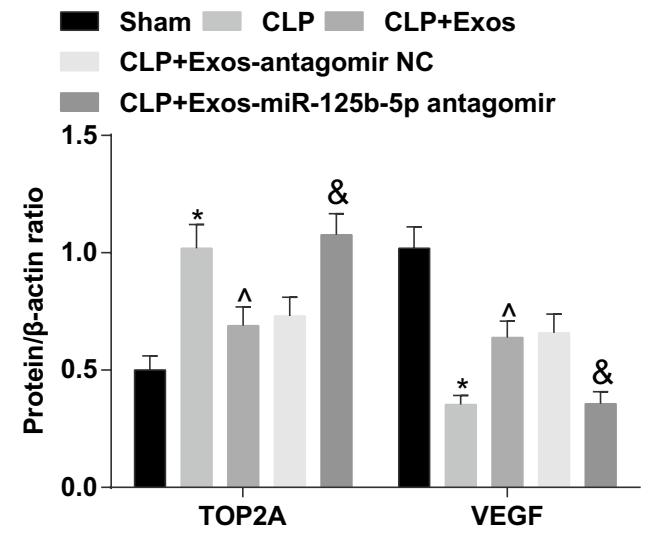

Fig. 4 Identification of EC-Exos, and EC-Exos promotes expression of miR-125b-5p and VEGF, while inhibiting expression of TOP2A in lung tissues of ALI mice. a Morphology of EC-Exos was observed under a TEM; b exosome marker proteins in serum were assessed by Western blot analysis; $\mathbf{c}$ effects of EC-Exos on the expression of miR$125 \mathrm{~b}-5 \mathrm{p}$, TOP2A and VEGF in lung tissues of mice; $\mathbf{d}$ protein bands of TOP2A and VEGF in lung tissues of mice with exosome treat-

Exos-antagomir NC, there showed approximate alveolar structure and less inflammatory cell infiltration in alveoli and pulmonary mesenchyme, while the inflammation reaction was still severer than the sham-operated mice.

TUNEL assay was further conducted to figure out the roles of exosomes on cell apoptosis in lung tissues of mice. Results (Fig. 6b) indicated that the number of apoptotic cells in lung tissues of ALI mice were decreased with EC-Exos treatment. EC-Exos conveying miR$125 b-5 p$ antagomir enhanced the number of apoptotic cells in mouse lung tissues. ment; e effects of EC-Exos on the protein expression of TOP2A and VEGF in lung tissues of mice; $* P<0.05$ vs the sham group, ${ }^{\wedge} P<0.05$ vs the CLP group, ${ }^{\&} P<0.05$ vs the CLP + Exos-antagomir NC group. The measurement data were expressed as mean \pm standard deviation, one-way ANOVA was used for comparisons among multiple groups and Tukey's post hoc test was used for pairwise comparisons after one-way ANOVA

\section{TOP2A is the target gene of miR-125b-5p}

It has been identified that TOP2A could act as a target gene of miRNA, which binds to the $3^{\prime} \mathrm{UTR}$ and regulate its expression. In order to confirm whether miR-125b-5p could target TOP2A, we firstly predicted using an online bioinformatic software (https://cm.jefferson.edu/), and it was found that there existed target relation between miR125b-5p and TOP2A (Fig. 7a). Results of dual luciferase reporter gene assay (Fig. 7b) suggested that the luciferase activity was apparently declined in ECs co-transfected with 
Fig. 5 EC-Exos decrease W/D value in lung tissues and protein content in BALF and also restrain inflammatory response in BALF and modulate VEGF expression in BALF and serum of ALI mice. a W/D values in lung tissues of mice; $\mathbf{b}$ protein contents in BALF; $\mathbf{c}$ concentrations of TNF- $\alpha$, IL- $1 \beta$ and IL- 6 in BALF; $\mathbf{d}$ VEGF expression in BALF and serum; $* P<0.05$ vs the sham group, $\wedge P<0.05$ vs the CLP group, ${ }^{\&} P<0.05$ vs the CLP+Exos-antagomir NC group. The measurement data were expressed as mean \pm standard deviation, one-way ANOVA was used for comparisons among multiple groups and Tukey's post hoc test was used for pairwise comparisons after one-way ANOVA
A

B

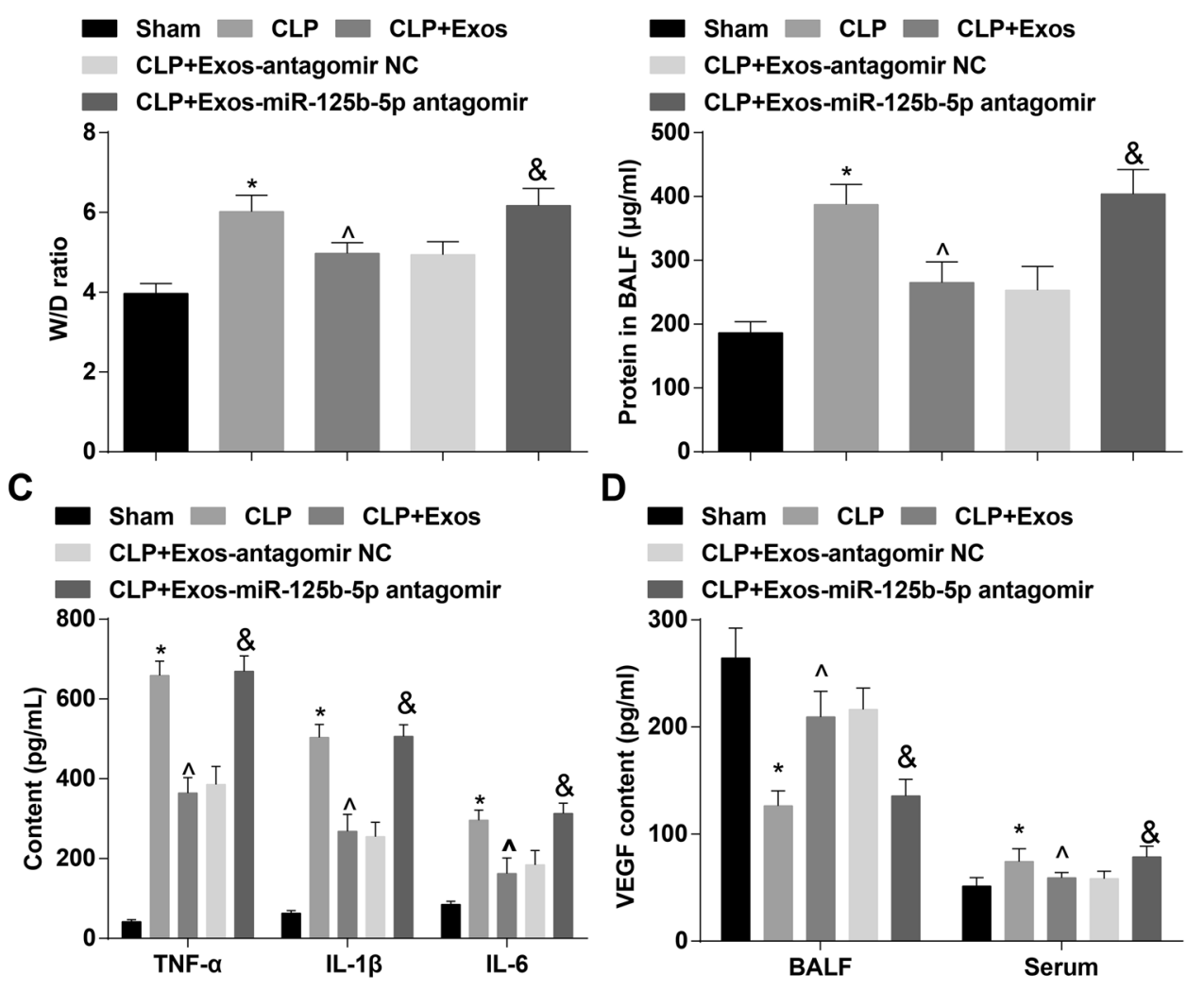

TOP2A-3'UTR-WT and miR-125b-5p mimics, while there was no evident difference in luciferase activity of ECs cotransfected with TOP2A-3'UTR-MUT and miR-125b-5p mimics.

\section{Discussion}

Affecting over 8 million people every year, sepsis is a fatal multiple-organ dysfunction. The lung is one of the most vulnerable organs to sepsis, which can develop into ALI and ARDS [20]. Exosomes represent major transport vehicles for miRNAs and exert the impact mostly by transferring miRNAs, thereby playing essential roles in physiology and diseases [14]. Our study focused on the role of exosomal miR-125b-5p in sepsis-induced ALI, and we have found that up-regulated exosomal miR-125b-5p were able to protect from sepsis-induced ALI by inhibiting TOP2A.

We first assessed miR-125b-5p, TOP2A and VEGF expression in ALI mice, and the results mirrored that miR125b-5p and VEGF were down-regulated but TOP2A was up-regulated in ALI mouse lung tissues. Similarly, Reithmair et al. have illuminated that miR-125b-5p was inhibited in non-survivors with sepsis [8], and Guo et al. have identified that miR-125b was down-regulated in BALF, peripheral blood and splenocytes from LPS-induced ALI mice [9]. It has also been proved that pulmonary VEGF expression was declined in ALI of a sepsis rat model [21]. As for the aberrant expression of TOP2A, Yang et al. have clarified that TOP2A was observed in the infected sepsis group rather than the control group [11], and a recent study has confirmed that the up-regulation of TOP2A has a potential relation in the progression of sepsis-related ARDS [12]. Moreover, we have discovered that there existed a target relation between miR-125b-5p and TOP2A in sepsis-induced ALI mice, which remains unexplored.

Another finding in this research suggested that the ECExos and elevated miR-125b-5p could decrease the levels of lung tissue water content and BALF protein. In line with this outcome, $\mathrm{Li}$ et al. have verified that mesenchymal stem cell-derived exosomal miR-124-3p reduced the W/D weight of lung tissues from rats with traumatic ALI [22], and it has been demonstrated that elevated miR-125b decreased the brain water content in rats with cerebral ischemia-reperfusion injury [23]. Additionally, we have found that the EC-Exos and the amplification of miR-125b-5p restrained inflammatory reaction in lung tissues of ALI mice. Consistently, Zhou et al. have illustrated that endothelial progenitor cell-derived exosomes could reduce inflammation in mice with LPS-induced ALI [24]. A recent study has verified 
A
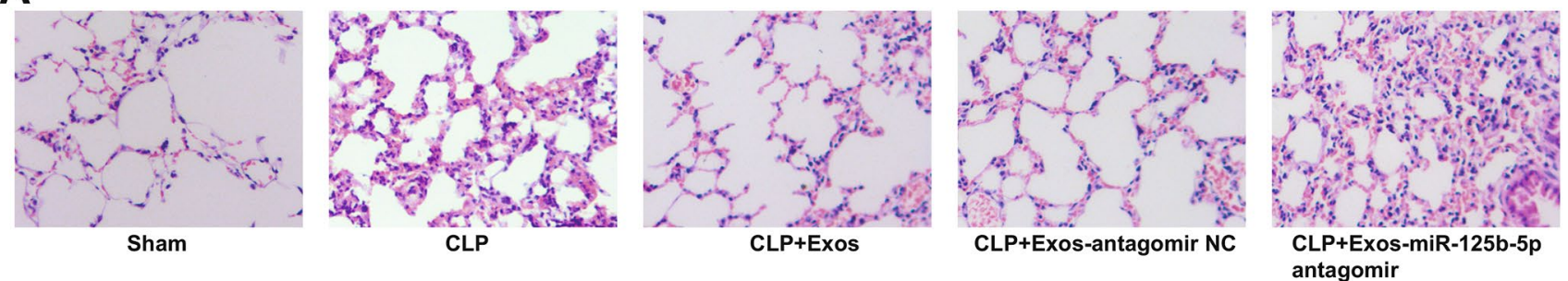

B
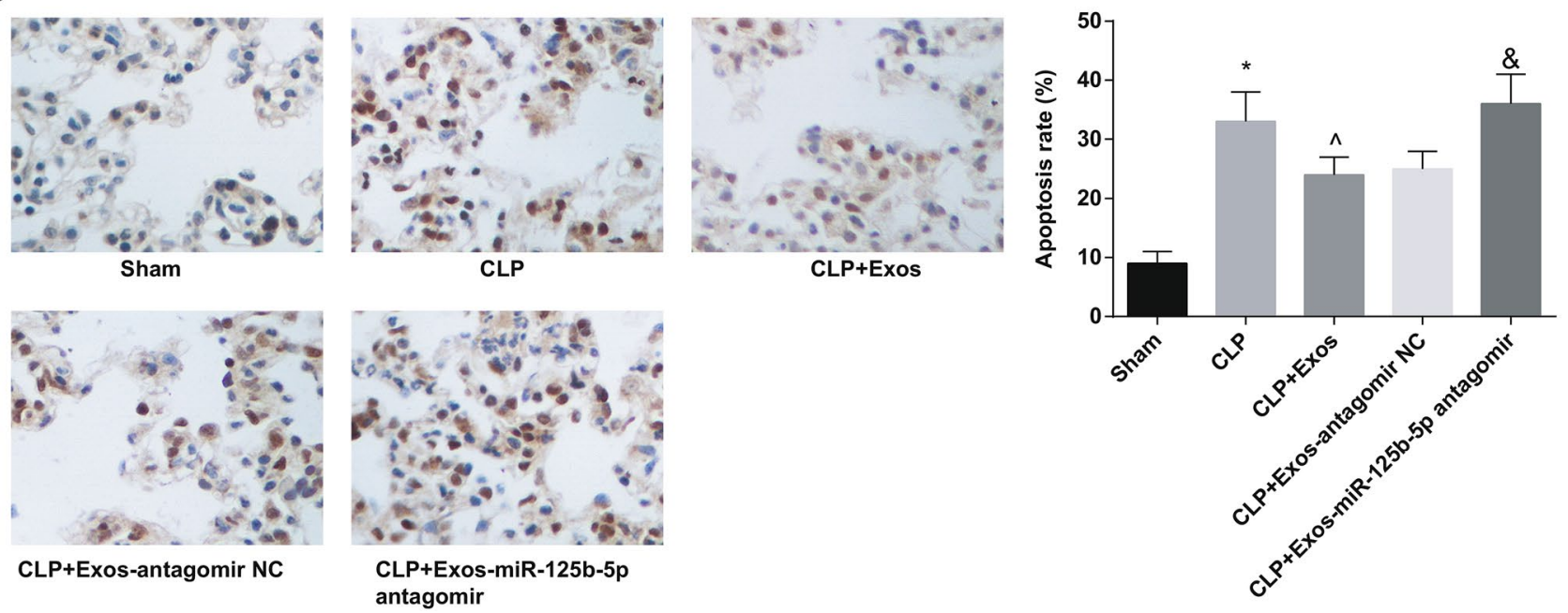

Fig. 6 EC-Exos improve pathological changes and decelerate cell apoptosis in lung tissues of ALI mice. a Pathological structure of lung tissues of mice; $\mathbf{b}$ representative images of TUNEL staining in lung tissues of mice and TUNEL positive cells in lung tissues of mice; $* P<0.05$ vs the sham group, ${ }^{\wedge} P<0.05$ vs the CLP group,

${ }^{\&} P<0.05$ vs the CLP + Exos-antagomir NC group. The measurement data were expressed as mean \pm standard deviation, one-way ANOVA was used for comparisons among multiple groups and Tukey's post hoc test was used for pairwise comparisons after one-way ANOVA

A

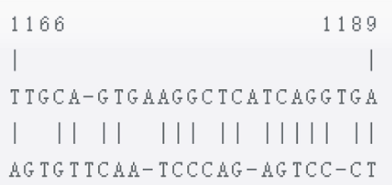

\begin{tabular}{|c|c|}
\hline $\begin{array}{l}\text { Gene Hane: Iop2a } \\
\text { ERSIUSG00000020914 } \\
\text { ERSIUST00000068031 }\end{array}$ & IRHA: $\quad$ nu-niR-125b-5p \\
\hline 1166 & 22 \\
\hline 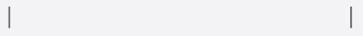 & 1 \\
\hline TTGCAGTGAAGGCTCATCAGGTGA & TCCCTGAGACCCTAACTTGTGA \\
\hline
\end{tabular}

Fig. 7 TOP2A is the target gene of miR-125b-5p. a Binding sites of miR-125b-5p and TOP2A were predicted by an online software; b target relation between miR-125b-5p and TOP2A was confirmed by dual luciferase reporter gene assay; $* P<0.01$ vs the mimic-NC
B

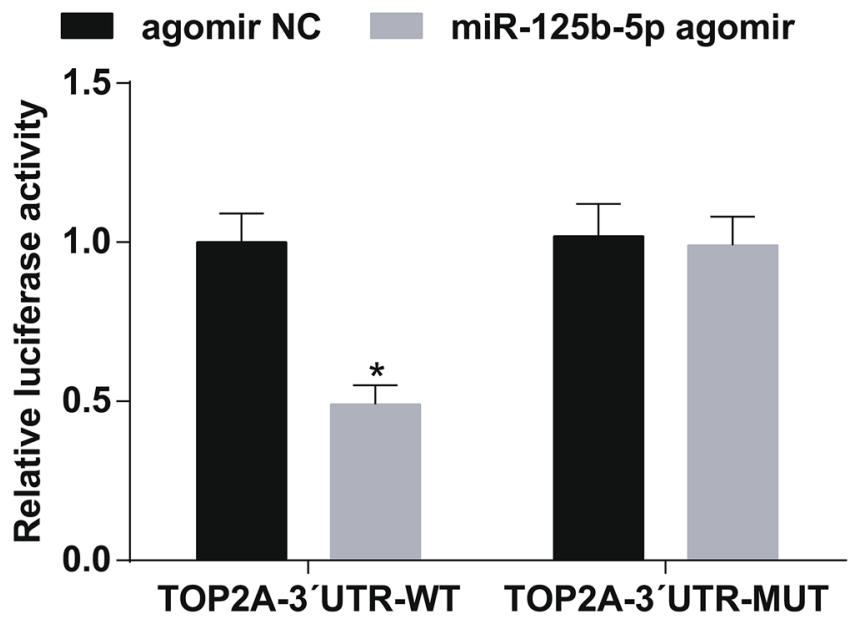

group. The measurement data conforming to the normal distribution were expressed as mean \pm standard deviation and the unpaired t-test was performed for comparisons between two groups 


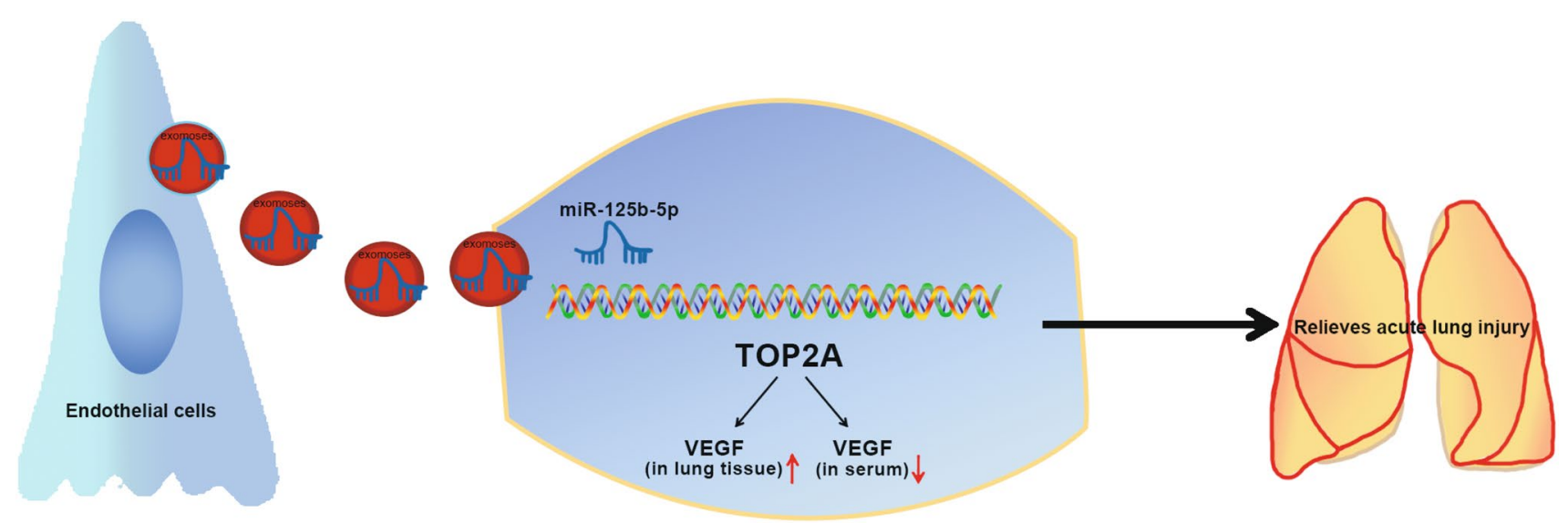

Fig. 8 The EC-derived exosomal miR-125b-5p inhibits TOP2A expression to suppress VEGF level, thus repressing apoptosis in lung tissue and ALI development

that overexpressed miR-125b-5p has the capacity to restrain inflammatory state in macrophages [25], and it has been figured out that the expression of TOP2A is concomitant with inflammation in human immunodeficiency virus (HIV)-1 infection [26]. Additionally, we have pointed out that the exosomes and amplified miR-125b-5p promoted VEGF in lung tissues while declined serum VEGF level in ALI mice. Consistently, Meng et al. have mentioned that overexpressed miR-125b decreased VEGF expression in liver sections from mice with cholestatic liver injury [27], and it has been recently unearthed that MSC-derived exosomes could decrease expression as well as secretion of VEGF in breast cancer cells [28]. Moreover, we have also discovered that the exosomes and exosomal miR-125b-5p restricted the cell apoptosis in ALI mouse lung tissues. In consistent with this finding, Huang et al. have proved that the MSC-derived exosomes were able to suppress oxygen-glucose deprivation-induced neuronal apoptosis [29]. A research has shown that overexpressed miR-125b-5p protected against cultured cardiomyocyte apoptosis in acute myocardial infarction [30], and Lin et al. have proved that TOP2A may contribute to apoptosis induction under isothiocyanate treatment [31].

In conclusion, we have demonstrated that up-regulation of ECs-derived exosomal miR-125b-5p protected against sepsis-induced ALI by inhibiting TOP2A (Fig. 8), which may provide novel targets for ALI treatment. Nevertheless, further research need to be conducted to reveal the detailed function mechanisms of exosomal miRNAs in sepsisinduced ALI.

Acknowledgements We would like to acknowledge the reviewers for their helpful comments on this paper.

Funding The current research was funded by Project of Shanghai Municipal Commission of Health and Family Planning (ZY(2018-2020)-FWTX-1108).

\section{Compliance with ethical standards}

Conflict of interest The authors declare that they have no conflicts of interest.

\section{References}

1. Sevransky JE, et al. Mortality in sepsis versus non-sepsis induced acute lung injury. Crit Care. 2009;13(5):R150.

2. Sadowitz B, et al. Lung injury induced by sepsis: lessons learned from large animal models and future directions for treatment. Expert Rev Anti Infect Ther. 2011;9(12):1169-78.

3. Gu WJ, et al. Risk of acute lung injury/acute respiratory distress syndrome in critically ill adult patients with pre-existing diabetes: a meta-analysis. PLoS ONE. 2014;9(2):e90426.

4. Aziz M, et al. B-1a cells protect mice from sepsis-induced acute lung injury. Mol Med. 2018;24(1):26.

5. Wang Y, et al. MicroRNA-31 regulating apoptosis by mediating the phosphatidylinositol-3 kinase/protein kinase B signaling pathway in treatment of spinal cord injury. Brain Dev. 2019;41(8):649-61.

6. Cao X, et al. MiR-145 negatively regulates TGFBR2 signaling responsible for sepsis-induced acute lung injury. Biomed Pharmacother. 2019;111:852-8.

7. Zhou J, et al. miR-206 regulates alveolar type II epithelial cell Cx43 expression in sepsis-induced acute lung injury. ExpTher Med. 2019a;18(1):296-304.

8. Reithmair M, et al. Cellular and extracellular miRNAs are bloodcompartment-specific diagnostic targets in sepsis. J Cell Mol Med. 2017;21(10):2403-11.

9. Guo Z, et al. Enforced expression of miR-125b attenuates LPSinduced acute lung injury. ImmunolLett. 2014;162(1 Pt A):18-26.

10. An X, et al. The prognostic significance of topoisomerase II alpha protein in early stage luminal breast cancer. BMC Cancer. 2018;18(1):331.

11. Yang J, Zhang P, Wang L. Gene network for identifying the entropy changes of different modules in pediatric sepsis. Cell PhysiolBiochem. 2016;40(5):1153-62.

12. Wang $M$, et al. Candidate genes and pathogenesis investigation for sepsis-related acute respiratory distress syndrome based on gene expression profile. Biol Res. 2016;49:25. 
13. Yamashita T, Takahashi Y, Takakura Y. Possibility of exosome-based therapeutics and challenges in production of exosomes eligible for therapeutic application. Biol Pharm Bull. 2018;41(6):835-42.

14. Wu X, et al. Exosomes derived from endothelial progenitor cells ameliorate acute lung injury by transferring miR-126. Exp Cell Res. 2018;370(1):13-23.

15. Yi X, et al. Exosomes derived from microRNA-30b-3p-overexpressing mesenchymal stem cells protect against lipopolysaccharide-induced acute lung injury by inhibiting SAA3. Exp Cell Res. 2019;383(2):111454.

16. Rittirsch $\mathrm{D}$, et al. Immunodesign of experimental sepsis by cecal ligation and puncture. Nat Protoc. 2009;4(1):31-6.

17. Zou Z, et al. Protective effects of P2X7R antagonist in sepsisinduced acute lung injury in mice via regulation of circ_0001679 and circ_0001212 and downstream Pln, Cdh2, and Nprl3 expression. J Gene Med. 2020. https://doi.org/10.1002/jgm.3261.

18. Mura M, et al. Vascular endothelial growth factor and related molecules in acute lung injury. J ApplPhysiol (1985). 2004;97(5):1605-17.

19. Papp A, et al. Decreased VEGF level is associated with elevated ferritin concentration in bronchoalveolar lavage fluid of children with interstitial lung diseases. Respiration. 2015;90(6):443-50.

20. Dong A, et al. Protective effects of hydrogen gas against sepsisinduced acute lung injury via regulation of mitochondrial function and dynamics. IntImmunopharmacol. 2018;65:366-72.

21. Jesmin S, et al. Time-dependent alterations of VEGF and its signaling molecules in acute lung injury in a rat model of sepsis. Inflammation. 2012;35(2):484-500.

22. Li QC, Liang Y, Su ZB. Prophylactic treatment with MSC-derived exosomes attenuates traumatic acute lung injury in rats. Am J Physiol Lung Cell MolPhysiol. 2019;316(6):L1107-17.

23. Xie YL, Zhang B, Jing L. MiR-125b blocks Bax/Cytochrome $\mathrm{C} / \mathrm{Caspase}-3$ apoptotic signaling pathway in rat models of cerebral ischemia-reperfusion injury by targeting p53. Neurol Res. 2018;40(10):828-37.
24. Zhou Y, et al. Exosomes from endothelial progenitor cells improve outcomes of the lipopolysaccharide-induced acute lung injury. Crit Care. 2019b;23(1):44.

25. Diao W, et al. MicroRNA-125b-5p modulates the inflammatory state of macrophages via targeting B7-H4. BiochemBiophys Res Commun. 2017;491(4):912-8.

26. Mandraju RK, Kondapi AK. Regulation of topoisomerase II alpha and beta in HIV-1 infected and uninfected neuroblastoma and astrocytoma cells: involvement of distinct nordihydroguaretic acid sensitive inflammatory pathways. Arch BiochemBiophys. 2007;461(1):40-9.

27. Meng F, et al. Regulation of the histamine/VEGF axis by miR$125 \mathrm{~b}$ during cholestatic liver injury in mice. Am J Pathol. 2014;184(3):662-73.

28. Pakravan K, et al. MicroRNA-100 shuttled by mesenchymal stem cell-derived exosomes suppresses in vitro angiogenesis through modulating the mTOR/HIF-1alpha/VEGF signaling axis in breast cancer cells. Cell Oncol (Dordr). 2017;40(5):457-70.

29. Huang $X$, et al. Exosomes derived from PEDF modified adiposederived mesenchymal stem cells ameliorate cerebral ischemiareperfusion injury by regulation of autophagy and apoptosis. Exp Cell Res. 2018;371(1):269-77.

30. Bayoumi AS, et al. A carvedilol-responsive microRNA, miR$125 \mathrm{~b}-5 \mathrm{p}$ protects the heart from acute myocardial infarction by repressing pro-apoptotic bak1 and klf13 in cardiomyocytes. J Mol Cell Cardiol. 2018;114:72-82.

31. Lin RK, et al. Dietary isothiocyanate-induced apoptosis via thiol modification of DNA topoisomerase IIalpha. J BiolChem. 2011;286(38):33591-600.

Publisher's Note Springer Nature remains neutral with regard to jurisdictional claims in published maps and institutional affiliations. 